\title{
CUMULATIVE AUTHOR INDEX
}

Aarseth, J.B., see Brevik

Abe, M. \& Nakanishi, N., Exact solutions to the two-dimensional BF and YangMills theories in the lightcone gauge

Abreu, E.M.C., Dalmazi, D. \& Silva, E.A., The Jacobi identity for Dirac-like brackets

Abrikosov, Jr. A.A., Fermion states on the sphere $S^{2}$

Actor, A., Vacuum response to electromagnetic backgrounds

Akant, L., Krishnaswami, G.S. \& Rajeev, S.G., Entropy of operator-valued random variables: A variational principle for large $N$ matrix models

Al-Aithan, T.A., see Riazuddin

Alhaidari, A.D., Exact solutions of Dirac and Schrödinger equations for a large class of power-law potentials at zero energy

Al-Naghmoush, A., Al-Thoyaib, S.S. \& Taha, M.O., Direct RG-improvement of the Higgs mass

Altenberger, A.R. \& Dahler, J.S., On the asymptotic behavior of the photon propagator in quantum electrodynamics

Al-Thoyaib, S.S., see AlNaghmoush

Ananthanarayan, B. \& Pasupathy, J., Higgs mass in the standard model from coupling constant reduction

Anazawa, M. \& Ishikawa, A., Geometrical construction of heterogeneous loop amplitudes in 2D gravity

Andersson, N., Gravitationalwave instabilities in rotating stars

Aoki, S., Experimental review of neutrino physics

Ardalan, F. \& Sadooghi, N., Anomaly and nonplanar diagrams in noncommutative gauge theories
A17 (2002) 776

A17 (2002) 1491

A17 (2002) 395

A17 (2002) 885

A17 (2002) 835

A17 (2002) 2413

A17 (2002) 4927

A17 (2002) 4551

A17 (2002) 685

A17 (2002) 279

A17 (2002) 685

A17 (2002) 335

A17 (2002) 4069

A17 (2002) 2645

A17 (2002) 3393

A17 (2002) 123
Arminjon, M., The scalar Ethertheory of gravitation and its first test in celestial mechanics

A17 (2002) 4203

Arnone, S., Kubyshin, Y.A., Morris, T.R. \& Tighe, J.F., Gauge-invariant regularisation via $\operatorname{su}(N \mid N)$

A17 (2002) 2283

Asano, M., Noncommutative branes in $D$-brane backgrounds

A17 (2002) 4733

Bagchi, B., Mallik, S. \& Quesne, C., Complexified PSUSY and SSUSY interpretations of some PT-symmetric Hamiltonians possessing two series of real energy eigenvalues

A17 (2002) 51

Bagneid, A.A., Constraints on neutral bosons of the $\operatorname{Sp}(6)_{L}$ $\otimes \mathrm{U}(1)_{Y}$ model

A17 (2002) 1273

Bagrov, V.G., Gitman, D.M. \& Tlyachev, V.B., L-dependence of particle radiation in magnetic-solenoid field as Aharonov-Bohm effect

A17 (2002) 1045

Bal, S. \& Takata, H., Interaction between two fuzzy spheres

A17 (2002) 2445

Balachandran, A.P. \& Digal, S., Topological string defect formation during the chiral phase transition

Balachandran, A.P. \& Roy, S.M., Continuous time-dependent measurements: Quantum anti-Zeno paradox with applications

Balbinot, R. \& Fabbri, A., 2D black holes and dimensional reduction

Baleanu, D. \& Güler, Y., The Hamilton-Jacobi treatment of front-form Schwinger model

Baleanu, D. \& Başkal, S., Dual metrics and nongeneric supersymmetries for a class of Siklos space-times

Bandeira de Melo, F.R.V., Brandenberger, R.H. \& Maia, A., Exponential growth of particle number far from the parametric resonance
A17 (2002) 4007

A17 (2002) 2745

A17 (2002) 1149

A17 (2002) 1449

A17 (2002) 3737

A17 (2002) 4413 
Banerjee, S., see Banerjee

Banerjee, S.N., Bhattacharya, A., Chakrabarti, B. \& Banerjee, S., On some properties of a meson

Banin, A.T. \& Pletnev, N.G., Application of symbol-operator technique

A17 (2002) 4939

A17 (2002) 4939

A17 (2002) 825

Barbieri, R., Quark masses and weak couplings in the SM and beyond

Barbón, J.L.F. \& Paredes, A., Noncommutative field theory and the dynamics of quantum Hall fluids

Barrabès, C. \& Hogan, P.A., Impulsive lightlike signals

Barton, G., Perturbative Casimir energies of spheres: Towards reorienting an agenda

Batista, A.B., Fabris, J.C., Goncalves, S.V.B. \& Tossa, J., Quantum perfect fluid cosmological model and its classical analogue

Battistel, O.A. \& Battistel, O.L., Anomalies in Ward Identities for three-point functions revisited

Battistel, O.L., see Battistel

Battiston, R., The Alpha magnetic spectrometer, a particle physics experiment in space

Battistoni, G., Ferrari, A. \& Sala, P.R., Calculation of secondary particles in atmosphere and hadronic interactions

Battye, R.A., Gravity and cosmology on a brane-world

Bayin, S., see Karaca

Başkal, S., see Baleanu

Beesham, A., Higher dimensional inhomogeneous dust collapse and cosmic censorship

Bengtsson, I., Brännlund, J. \& Życzkowskí, K., CP ${ }^{n}$, or, entanglement illustrated

Berezin, V., Towards a theory of quantum black holes

Bergqvist, G., Causal tensors and simple forms

Bertucci, B., Review of precision measurements of high energy electrons

A17 (2002) 3589

A17 (2002) 2746

A17 (2002) 767

A17 (2002) 1979

A17 (2002) 1979

A17 (2002) 1589

A17 (2002) 1743

A17 (2002) 2651

A17 (2002) 3737

A17 (2002) 2747

A17 (2002) 4675

A17 (2002) 979

A17 (2002) 2748

A17 (2002) 1613
Bezerra de Mello, E.R. \& Carvalho, F.C., Vacuum polarization in the global monopole spacetime at finite temperature

A17 (2002) 879

Bezerra de Mello, E.R., see Spinelly

Bezerra, V.B., de A. Marques, G. \& Khusnutdinov, N.R., Some remarks on topological defects and their gravitational consequences

Bezerra, V.B., see Khusnutdinov

Bhattacharya, A., see Banerjee Bhattacharyya, S., Kumar, A. \& Mukhopadhyay, S., Curved membrane solutions in $D=$ 11 supergravity

Bhattacharyya, D.P., see Guptaroy

Bhattacharyya, S., see Guptaroy

Bhattacharyya, S., see De

Bichl, A.A., Grimstrup, J.M., Popp, L., Schweda, M. \& Wulkenhaar, R., Perturbative analysis of the SeibergWitten map

A17 (2002) 4457
Bigi, I. \& Uraltsev, N., On the expected photon spectrum in $B \rightarrow X_{\mathrm{s}}+\gamma$ and its uses

Bijnens, J., Chiral Lagrangians Birmingham, D., Choptuik scaling and quasinormal modes in the AdS/CFT correspondence

Bizdadea, C., Cioroianu, E.M. \& Saliu, S.O., Hamiltonian cohomological derivation of four-dimensional nonlinear gauge theories

Bohm, A., see Kaldass

Boisseau, B., Perturbations of a cylindrical vortex in a relativistic perfect isentropic fluid and Nambu-Goto dynamics

Bonazzola, S., see Gourgoulhon

Bonazzola, S., see Villain

Bordag, M. \& Kirsten, K., Heat kernel coefficients and divergencies of the Casimir energy for the dispersive sphere

Bossi, F., Status of the KLOE experiment at DAФNE
A17 (2002) 4375

A17 (2002) 4365

A17 (2002) 870

A17 (2002) 4939

A17 (2002) 4647

A17 (2002) 1159

A17 (2002) 1159

A17 (2002) 4615

A17 (2002) 2219

A17 (2002) 4709

A17 (2002) 3154

A17 (2002) 2750

A17 (2002) 2191

A17 (2002) 3749

A17 (2002) 2751

A17 (2002) 2689

A17 (2002) 2780

A17 (2002) 813

A17 (2002) 3026 
Brandenberger, R.H. \& Martin, J., On signatures of short distance physics in the cosmic microwave background

Brandenberger, R.H., see Bandeira

Brännlund, J., see Bengtsson

Brautti, G. \& Picca, D., Thermal stimulation of gravitational wave antennas

Brautti, G. \& Picca, D., The sensitivity of antennas for gravitational wave detection

Brevik, I., Aarseth, J.B. \& Høye, J.S., Casimir problem in spherical dielectrics: A quantum statistical mechanical approach

Bruni, M., see Mena

Buénerd, M., Simulation of atmospheric secondary hadron and lepton flux from satellite to underground experiments

Bulgac, A., see Magierski

Burgess, C.P., Matias, J. \& Pospelov, M., A Higgs or not a Higgs? What to do if you discover a new scalar particle

Burinskii, A., Casimir energy and vacua for superconducting ball in supergravity

Bussey, P.J., Heavy flavour physics at HERA - a survey

Bytsenko, A.A. \& Goncharov, Yu.P., Dirac monopoles and Hawking radiation in Kottler spacetime

Bytsenko, A.A., Elizalde, E. \& Sukhanov, S.A., Hyperbolic topological invariants and the black hole geometry

Bytsenko, A.A., Chaves, F.M.P. \& Gonçalves, A.E., Nonplanar contribution to the effective action in noncommutative flat manifolds

Bytsenko, A.A., Determinant line bundles and topological invariants of hyperbolic geometry

Cabibbo, N., Concluding remarks

Calloni, E., Di Fiore, L., Esposito, G., Milano, L. \& Rosa, L., Gravitational effects on a rigid Casimir cavity
A17 (2002) 3663

A17 (2002) 4413

A17 (2002) 4675

A17 (2002) 1111

A17 (2002) 327

A17 (2002) 776

A17 (2002) 4239

A17 (2002) 1665

A17 (2002) 1059

A17 (2002) 1841

A17 (2002) 920

A17 (2002) 1065

A17 (2002) 4947

A17 (2002) 4167

A17 (2002) 4349

A17 (2002) 951

A17 (2002) 3500

A17 (2002) 804
Calogeracos, A., Radiation from perfect mirrors following prescribed relativistic trajectories

Canal, C.A.G., see Daleo

A17 (2002) 1018

A17 (2002) 269

Cardoso, V. \& Lemos, J.P.S., Quasinormal modes of Schwarzschild-anti-de Sitter black holes: Electromagnetic and gravitational perturbations

A17 (2002) 2752

A17 (2002) 2767

Cardoso, V., see Lemos

Carli, T., Small-X QCD effects in particle collisions at high energies

A17 (2002) 3185

Carmeli, M., Fundamental approach to the cosmological constant issue

Carvalho, F.C., see Bezerra de Mello

Casadio, R. \& Harms, B., Can black holes and naked singularities be detected in accelerators?

Casadio, R., Gamma-ray bursts from gravitational collapse

Casana, R. \& Dias, S.A., Exact renormalization of massless $\mathrm{QED}_{2}$

Casana, R., Pimentel, B.M., Lunardi, J.T. \& Teixeira, R.G. Free electromagnetic field in Riemannian space-time via DKP theory

A17 (2002) 4197

Casaus, J., Review on precision measurements of high energy hadrons

Casolino, M., Low energy solar and galactic cosmic rays at 1 AU

Cassel, D.G., CLEO results: $B$ decays

Casteill, P.Y. \& Valent, G., New self-dual Einstein metrics

Cei, F., Neutrinos from supernovae: Experimental status

Chakrabarti, B., see Banerjee

Chang, Z. \& Guan, C.-B., Dynamics of massive scalar fields in $\mathrm{dS}$ space and the dS/ CFT correspondence

A17 (2002) 4219

A17 (2002) 879

A17 (2002) 4635

A17 (2002) 2753

A17 (2002) 4601

A17 (2002) 1603

A17 (2002) 1685

A17 (2002) 2951

A17 (2002) 2754

A17 (2002) 1765

A17 (2002) 4939

Chankowski, P.H. \& Pokorski, S., Quantum corrections to neutrino masses and mixing angles 
Chaves, F.M.P., see Bytsenko

Chen, F., Harris, B.W., Roy, A. \& Mohideen, U., Measurements of the normal and shape dependent Casimir forces using an atomic force microscope

Chen, G., see Zhang

Chen, J.-S., Li, J.-R. \& Zhuang, P.-F., Non-Abelian medium effects in quark-gluon plasma

Cherednikov, I.O., Casimir energy of confined fields: A role of the RG-invariance

Chervon, S.V., Cosmological models of global universe evolution and decomposition of perturbations

Chervyakov, A., see Kleinert

Cheshkov, C., Nonleptonic kaon decays: Theory vs. experiment

Choudhury, A.G. \& Chowdhury, A.R., Discrete Kaup-Newell equation, canonical Bäcklund transformation and Baxter's $Q$-operator

Choutko, V., Lamanna, G. \& Malinin, A., Cosmic photon and positron spectra measurements modelling with the AMS-02 detector at ISS

Chowdhury, A.R., see Choudhury

Cieza Montalvo, J.E. \& De Queiroz Filho, P.P., Pair of heavy-exotic-quarks at LHC

Cioroianu, E.M., see Bizdadea

Close, F.E., Light hadron spectroscopy

Cocoletzi, G.H., see Villarreal

Cohen, T.D. \& Glozman, L.Ya., Does one observe chiral symmetry restoration in baryon spectrum?

Coley, A.A. \& van den Hoogen, R.J., Assisted inflation

Colistete Jr., R., Higher-order geodesic deviations and the calculus of relativistic orbits

Cordero, R. \& Rojas, E., Chiral superconducting membranes

Curado, E.M.F., see RegoMonteiro

da Silva, A.J., see Girotti
A17 (2002) 4349

A17 (2002) 711

A17 (2002) 4543

A17 (2002) 1435

A17 (2002) 874

A17 (2002) 4451

A17 (2002) 2019

A17 (2002) 3827

A17 (2002) 515

A17 (2002) 1817

A17 (2002) 515

A17 (2002) 4133

A17 (2002) 2191

A17 (2002) 3239

A17 (2002) 798

A17 (2002) 1327

A17 (2002) 2755

A17 (2002) 2756

A17 (2002) 73

A17 (2002) 661

A17 (2002) 1503
Dahler, J.S., see Altenberger

Daleo, A., Canal, C.A.G., Navarro, G.A. \& Sassot, R., On the $\bar{d} / \bar{u}$ asymmetry and parton distributions

Dalmazi, D., see Abreu

Damião Soares, I., see de Oliveira

Damour, T., Chaos in string cosmology

Darewych, J.W., see Di Leo

de A. Marques, G., see Bezerra

De, B. see Guptaroy

de Oliveira, H.P. \& Damião Soares, I., A dynamical system approach to critical gravitational collapse

A17 (2002) 4177

De Queiroz Filho, P.P., see Cieza Montalvo

de S. Pires, C.A., Inflation in extra dimensions

de Sousa, C.A., see Jaminon

De, B., Bhattacharyya, S. \& Guptaroy, P., Studies in rapidity and $p_{T}$-spectra of pions in high energy NN, NA and AA collisions: A comprehensive approach

Debergh, N., Van den Bossche, B. \& Samsonov, B.F., Darboux transformations for quasiexactly solvable Hamiltonians

Del Popolo, A., Gambera, M. \& Ercan, E.N., Dynamical evolution of clusters of galaxies: the effect of high-velocity substructure clumps

Deser, S., New properties of matter in (A)dS and their consequences

Di Fiore, L., see Calloni

Di Leo, L. \& Darewych, J.W., A variational Fock-space treatment of quarkonium

Diamandis, G.A., Georgalas, B.C., Mavromatos, N.E., Papantonopoulos, E. \& Pappa, I., Cosmological evolution in a type-0 string theory

Diamandis, G.A., Georgalas, B.C., Mavromatos, N.E. \& Papantonopoulos, E., Acceleration of the universe in type-0 noncritical strings
A17 (2002) 279

A17 (2002) 269

A17 (2002) 395

A17 (2002) 4177

A17 (2002) 2655

A17 (2002) 2165

A17 (2002) 4365

A17 (2002) 1159

A17 (2002) 4133

A17 (2002) 4335

A17 (2002) 4903

A17 (2002) 4615

A17 (2002) 1577

A17 (2002) 187

A17 (2002) 2661

A17 (2002) 804

A17 (2002) 2165

A17 (2002) 2241

A17 (2002) 4567 
Dias, S.A., see Casana

Digal, S., see Balachandran

Djordjević, G.S., Dragovich, B., Nešić, L.D. \& Volovich, I.V., $p$-adic and adelic minisuperspace quantum cosmology

Dorfan, J., Babar results on $\mathrm{CP}$ violation

Downes, P., see Robinson

Dragovich, B., see Djordjević

Drees, J., Review of final LEP results or a tribute to LEP

Dunne, G.V. \& Schubert, Ch., Two-loop Euler-Heisenberg Lagrangians and Borel analysis

Dunne, G.V., Finite temperature induced fermion number

Durante, M., Biological effects of cosmic radiation in lowearth orbit

Edgar, B. \& Ludwig, G., Finding conformal Killing vectors from the invariant classification scheme

Elizalde, E., see Bytsenko

Ellis, G.F.R., Cosmology and local physics

Ellis, J., Decline and fall of the Standard Model?

Ercan, E.N., see Del Popolo

Ercolessi, E., Morandi, G. \& Marmo, G., Alternative Hamiltonian descriptions and statistical mechanics

Erdmann, M., Proton and photon structure

Esposito, G., see Fiandrini

Esposito, G., see Calloni

Esposito, G., A spectral approach to Yang-Mills theory

Esquivel-Sirvent, R., see Villarreal

Fabbri, A., see Balbinot

Fabris, J. \& Spindel, Ph., Scalar perturbations in a primordial inflationary scenario

Fabris, J.C., see Batista

Fagundes, H.V., see Müller

Fakhri, H. \& Motavali, H., Parasupersymmetric coherent states for Landau levels with dynamical symmetry group $\mathrm{H}_{4}$
A17 (2002) 4601

A17 (2002) 1149

A17 (2002) 1413

A17 (2002) 2925

A17 (2002) 2733

A17 (2002) 1413

A17 (2002) 3259

A17 (2002) 956

A17 (2002) 890

A17 (2002) 1713

A17 (2002) 2757

A17 (2002) 4167

A17 (2002) 2667

A17 (2002) 3284

A17 (2002) 187

A17 (2002) 3779

A17 (2002) 3171

A17 (2002) 1655

A17 (2002) 804

A17 (2002) 926

A17 (2002) 798

A17 (2002) 2745

A17 (2002) 2758

A17 (2002) 2749

A17 (2002) 4385

A17 (2002) 4081
Falcone, D., Fermion masses and mixings in gauge theories

Fan, H.-Y. \& Fan, Y., Weyl ordering for entangled state representation

Fan, H.-Y., Bose operator Hamiltonian model for rotating electric dipole and generalized Ehrenfest's theorem

Fan, Y., see Fan

Farina, C., Santos, F.C. \& Tort, A.C., On the dispersive van der Waals forces

Feinberg, J., The periodic table of static fermion bags in the Gross-Neveu model

A17 (2002) 3981

A17 (2002) 701

A17 (2002) 45

A17 (2002) 701

A17 (2002) 794

A17 (2002) 898

Felder, G., Warped geometry of brane worlds

Félix-Beltrán, O., Higgs masses and coupling within an extension of the MSSM with Higgs triplets

Ferrari, A., see Battistoni

Fiandrini, E. \& Esposito, G., Leptons with E > $200 \mathrm{Mev}$ trapped in the South Atlantic Anomaly

Florides, P.S., Einstein's equivalence principle and the gravitational red shift

Flórez, J.B., see Ponce

Fonseca, J., The equivalence problem in teleparallel gravity

Ford, L.H. \& Svaiter, N.F., The focusing of vacuum fluctuations

Freyhult, L., Field decomposition and the ground state structure of SU(2) YangMills theory

A17 (2002) 3681

Frolov, V., Cosmic strings and energy mining from black holes

A17 (2002) 2673

Fry, M.P., Fermion determinants

Futamase, T., Observations of dark side of the universe by gravitational lensing

A17 (2002) 465

A17 (2002) 1743

A17 (2002) 1655

A17 (2002) 2759

A17 (2002) 643

A17 (2002) 4161

A17 (2002) 4393

A17 (2002) 936

A17 (2002) 2677

Gabadadze, G. \& Shifman, M., QCD vacuum and axions: What's happening?

A17 (2002) 3689

Gaeta, G., Poincaré normal forms and simple compact Lie groups

A17 (2002) 3571 
Gagnon, P., Production, spectroscopy and decay of orbitally excited $B$ mesons

Gambera, M., see Del Popolo

Gamberg, L., see Milton

Gamboa, J., Méndez, F., Loewe, M. \& Rojas, J.C., Noncommutative quantum mechanics: The two-dimensional central field

Gangui, A., Early universe sources for CMB nonGaussianity

Garattini, R., Entropy from the foam II

Garattini, R., Wormholes and space-time foam: The case with a cosmological constant

Garattini, R., Space time foam

Gato-Rivera, B., Construction formulae for singular vectors of the topological and of the Ramond $N=2$ superconformal algebras

Gauthier, C., Gravel, P. \& Melanson, J., New lower bounds for warp drive energy

Geer, S., Neutrino factory and muon collider R\&D

Genet, C., Lambrecht, A. \& Reynaud, S., Correlation between plasma and temperature corrections to the Casimir force

Georgalas, B.C., see Diamandis Georgalas, B.C., see Diamandis Geyer, B. \& Mülsch, D., Hodge type cohomological gauge theories

Geyer, B., Lavrov, P. \& Nersessian, A., Integration measure and extended BRST-covariant quantization

Gibilisco, M., An analytical solution of the cosmic rays transport equation in the presence of the geomagnetic field

Gies, H. \& Langfeld, K., Loops and loop clouds - A numerical approach to the worldline formalism in QED

Gilani, A.H.S., see Riazuddin

Giribet, G. \& Simeone, C., Deparametrization and quantization of the Taub universe
A17 (2002) 145

A17 (2002) 187

A17 (2002) 732

A17 (2002) 2555

A17 (2002) 4273

A17 (2002) 1965

A17 (2002) 2760

A17 (2002) 829

A17 (2002) 4515

A17 (2002) 2761

A17 (2002) 3483

A17 (2002) 761

A17 (2002) 2241

A17 (2002) 4567

A17 (2002) 4425

A17 (2002) 1183

A17 (2002) 1645

A17 (2002) 966

A17 (2002) 4927

A17 (2002) 2885
Girotti, H.O., Gomes, M., Rivelles, V.O. \& da Silva, A.J., The noncommutative supersymmetric nonlinear sigma model

A17 (2002) 1503

Gitman, D.M., see Bagrov

Glozman, L.Ya., see Cohen

Gnedin, Y.N., Current status of modern dark matter problem

Golshani, M., see Motavali

Gomero, G.I., Rebouças, M.J. \& Tavakol, R., Limits on the detectability of cosmic topology in hyperbolic universes

Gomero, G.I., Can we see the shape of our universe?

Gomes, M., see Girotti

Gonçalves, A.E., see Bytsenko

Goncalves, S.V.B., see Batista

Goncharov, Yu.P., see Bytsenko

Goodman, J.A., Recent results from Super-Kamiokande

Gourgoulhon, E. \& Novak, J., Covariant conformal decomposition of Einstein equations

Gourgoulhon, É., Grandclément, P. \& Bonazzola, S., Last orbits of binary black holes

Graham, N., Jaffe, R.L. \& Weigel, H., Casimir effects in renormalizable quantum field theories

Grandclément, P., see Gourgoulhon

Gravel, P., see Gauthier

Grib, A.A. \& Pavlov, Yu.V., Cold dark matter and primordial superheavy particles

Grimstrup, J.M., see Bichl

Grosse, H., Maceda, M., Madore, J. \& Steinacker, H., Fuzzy instantons

Grumiller, D., The virtual black hole in $2 \mathrm{D}$ quantum gravity and its relevance for the Smatrix

Guan, C.-B., see Chang

Gubarev, F.V. \& Zakharov, V.I., The Berry phase and monopoles in non-Abelian gauge theories

A17 (2002) 1045

A17 (2002) 1327

A17 (2002) 4251

A17 (2002) 375

A17 (2002) 4261

A17 (2002) 4281

A17 (2002) 1503

A17 (2002) 4349

A17 (2002) 2749

A17 (2002) 4947

A17 (2002) 3353

A17 (2002) 2762

A17 (2002) 2689

A17 (2002) 846

A17 (2002) 2689

A17 (2002) 2761

A17 (2002) 4435

A17 (2002) 2219

A17 (2002) 2095

A17 (2002) 989

A17 (2002) 4591

Guendelman,E.I.\& Kaganovich, A.B., SSB of scale symmetry, fermion families and quintessence without the long-range force problem 
Guendelman, E.I. \& Kaganovich, A.B., Quintessential potential, fermion families and spontaneous breaking of scale symmetry

Guha, P., Generalized Poisson mechanics in $D$-branes

Guimarães, M.E.X., String's current induced by the dilatonic coupling of gravity

Güler, Y., see Baleanu

Gupta, M., see Randhawa

Guptaroy, P., Bhattacharyya, D.P., De, B. \& Bhattacharyya, S., Nature of antiproton production in high energy nuclear collisions: Assorted data versus specific models

Guptaroy, P., see De

Gustavsson, A., The $d=6$, $(2,0)$-tensor multiplet coupled to self-dual strings

Gustavsson, A., On the holomorphically factorized partition function for Abelian gauge theory in six dimensions

Haba, Z. \& Kleinert, H., Quantum-Liouville and Langevin equations for gravitational radiation damping

Hall, R.L., Lucha, W. \& Schöberl, F.F., Discrete spectra of semirelativistic Hamiltonians from envelope theory

Halliwell, J.J., see Thorwart

Halpern, M.B. \& Thorn, C.B., Large $N$ matrix mechanics on the light-cone

Halpern, M.B. \& Obers, N.A., Two large examples in orbifold theory: Abelian orbifolds and the charge conjugation orbifold on $\mathfrak{s u}(n)$

Halzen, F., The highest energy cosmic rays, gamma-rays and neutrinos: Facts, fancy and resolution

Handrich, G., Lorentz covariance for the quantum algebra of observables: NambuGoto strings in $3+1$ dimensions

Hanson, G.G., Searches for new particles
Harikumar, E. \& Sivakumar, M., Hamiltonian vs Lagrangian embedding of a massive spinone theory involving two-

A17 (2002) 1159

A17 (2002) 4615

A17 (2002) 2051

A17 (2002) 383

A17 (2002) 3729

A17 (2002) 1931

A17 (2002) 2778

A17 (2002) 1517

A17 (2002) 3897

A17 (2002) 3432

A17 (2002) 2331

A17 (2002) 3336 form field

Harms, B., see Casadio

Harris, B.W., see Chen

Heenen, P.-H., see Magierski

Hehl, F.W., Obukhov, Y.N. \& Rubilar, G.F., Light propagation in generally covariant electrodynamics and the Fresnel equation

Herrera-Aguilar, A. \& Kechkin, O.V., String theory extensions of Einstein-Maxwell fields: The static case

Heuer, R.-D., Electron-positroncolliders

Heynderickx, D., Review on modelling of the radiation belts

Hlavatý, L., see Šnobl

Hogan, P.A., see Barrabès

Høye, J.S., see Brevik

Huey, G. \& Tavakol, R., Quintessence tracking in generalised settings

Iacobucci, G., Diffractive phenomena

Iga, K., What do topologists want from Seiberg-Witten theory?

Iliev, B.Z., Fibre bundle formulation of nonrelativistic quantum mechanics

iv. mixed states and evolution transport's curvature

A17 (2002) 229

Iliev, B.Z., Fibre bundle formulation of nonrelativistic quantum mechanics

v. interpretation, summary and discussion

A17 (2002) 245

Iorio, A. \& Sýkora, T., On the space-time symmetries of noncommutative gauge theories

Ishikawa, A., see Anazawa

Isidori, G., Rare decays: Theory vs. experiments

Israelit, M., Weyl-Dirac quintessence and dark matter

A17 (2002) 405

A17 (2002) 4635

A17 (2002) 711

A17 (2002) 1059

A17 (2002) 2695

A17 (2002) 2485

A17 (2002) 3469

A17 (2002) 1675

A17 (2002) 4043

A17 (2002) 2746

A17 (2002) 776

A17 (2002) 4403

A17 (2002) 3204

A17 (2002) 4463

Itin, Y., Conserved current for general teleparallel models Itin, Y., see Kaniel
A17 (2002) 2369

A17 (2002) 4069

A17 (2002) 3078

A17 (2002) 4229

A17 (2002) 2765

A17 (2002) 2766 
Ivezić, T., see Škovrlj

Jackson, H.E., Hermes and the spin of the proton

Jackson, J.D., Criticism of "Necessity of simultaneous coexistence of instantaneous and retarded interactions in classical electrodynamics" by Chubykalo and Vlaev

Jaekel, M.-T., see Reynaud

Jaffe, R.L., see Graham

Jain, A., Jain, P., McKay, D.W. \& Ralston, J.P., Graviton enhanced UHE neutrino cross-sections and giant air showers

Jain, P., see Jain

Jaminon, M., Ruivo, M.C. \& de Sousa, C.A., Pion and $\rho$ meson observables in a Nambu-Jona-Lasinio model including a tensor interaction

Jiong, S., see Sirendaoreji

Joglekar, S.D. \& Mandal, B.P., Application of finitefield-dependent BRS transformations to problems of the Coulomb gauge

Jung, C.K., Recent results from $\mathrm{K} 2 \mathrm{~K}$

Kagali, B.A., see Rao

Kaganovich, A.B., see Guendelman

Kaganovich, A.B., see Guendelman

Kakushadze, Z., On gauge dynamics and SUSY breaking in orientiworld

Kalbfleisch, G.R., see Milton

Kaldass, H., Bohm, A. \& Wickramasekara, S., Resonance states from poles of the relativistic $S$-matrix

Kaniel, S. \& Itin, Y., On the derivation of the equations of motion

Karaca, K. \& Bayin, S., An open singularity-free cosmological model with inflation

Kavic, M., Matching weak coupling and quasiclassical expansions for dual QES problems

Kechkin, O.V., see HerreraAguilar
A17 (2002) 2513

A17 (2002) 3551

A17 (2002) 3975

A17 (2002) 1003

A17 (2002) 846

A17 (2002) 533

A17 (2002) 533

A17 (2002) 4903

A17 (2002) 2897

A17 (2002) 1279

A17 (2002) 3364

A17 (2002) 4793

A17 (2002) 417

A17 (2002) 4419

A17 (2002) 3875

A17 (2002) 732

A17 (2002) 3749

A17 (2002) 2766

A17 (2002) 4457

A17 (2002) 4661

A17 (2002) 2485
Kessler, R., Recent KTEV results

A17 (2002) 2999

Khanna, F.C., see Zhao

A17 (2002) 2911

Khusnutdinov, N.R. \& Bezerra, V.B., Self-energy in the GottHiscock space-time

A17 (2002) 870

Khusnutdinov, N.R., see Bezerra

Kim, Y.-K., Tevatron: Present status and future prospects

Kirsten, K., see Bordag

Kitabayashi, T. \& Yasuè, M., Large solar neutrino mixing in an extended Zee model

Klapdor-Kleingrothaus, H.V., Dark matter search

A17 (2002) 4365

A17 (2002) 3099

A17 (2002) 813

A17 (2002) 2519

A17 (2002) 3421

Klein, J.R., Solar neutrino results from the Sudbury Neutrino Observatory

A17 (2002) 3378

Kleinert, H. \& Chervyakov, A., Integrals over products of distributions from perturbation expansions of path integrals in curved space

Kleinert, H., see Haba

Klich, I., Casimir energy of a dielectric-diamagnetic media

Klimchitskaya, G.L. \& Mohideen, U., Constraints on Yukawa-type hypothetical interactions from recent Casimir force measurements Klimchitskaya, G.L., What is the temperature dependence of the Casimir force between real metals?

Kniehl, B.A., Theoretical aspects of Standard-Model Higgs-Boson physics at a future $e^{+} e^{-}$linear collider

Kogan, I.I. \& Nichols, A., $\mathrm{SU}(2)_{0}$ and $\operatorname{OSp}(2 \mid 2)_{-2}$ WZNW models: Two current algebras, one logarithmic CFT

Kojima, T., The affine $A_{n-1}^{(1)}$ Toda fields with boundary reflection

Konar, S., Photon propagation in a magnetized medium

Kovner, A., Magnetic $Z_{N}$ symmetry in $2+1$ dimensions

Krishnaswami, G.S., see Akant

Kubyshin, Y.A., see Arnone

Kubyshin, Yu.A., Neves, R. \& Potting, R., Solutions of the
A17 (2002) 2615

A17 (2002) 487

A17 (2002) 2019

A17 (2002) 3729

A17 (2002) 808

A17 (2002) 751

A17 (2002) 1457

A17 (2002) 1055

A17 (2002) 2113

A17 (2002) 2413

A17 (2002) 2283
A17 (2002) 4143 
Polchinski ERG equation in the $O(N)$ scalar model

Kumar, A., see Bhattacharyya

Kunzinger, M., see Steinbauer

Lachièze-Rey, M., see Mbelek

Lamanna, G., see Choutko

Lambiase, G., see Nesterenko

Lambrecht, A., see Reynaud

Lambrecht, A., see Genet

Lamine, B., see Reynaud

Langfeld, K., see Gies

Langlois, D., Gravitational and cosmological properties of a brane-universe

Lanz, L. \& Vacchini, B., Subdynamics of relevant observables: A field theoretical approach

Lauscher, O. \& Reuter, M., Towards nonperturbative renormalizability of quantum Einstein gravity

Lavrov, P., see Geyer

Lee, B.J. \& Lee, T.H., Scalar nonluminous matter in galaxies

Lee, T.H., see Lee

Legaré, M., Solutions of vanishing curvature non-Abelian monopole equations

Lemos, J.P.S. \& Cardoso, V., Radiation generated by the infall of a scalar particle in a Schwarzschild-anti-de Sitter background

Lemos, J.P.S., see Cardoso

Li, B.A., High energy behavior of the SM at unitary gauge

Li, J.-R., see Chen

Liao, H.-B. \& Wu, Y.-F., On the stability of rapidity gap analysis

Liu, L.-S., see Zhang

Loewe, M., see Gamboa

Loran, F. \& Shirzad, A., Classification of constraints using the chain-by-chain method

Loran, F., see Shirzad

Lucha, W. \& Schöberl, F.F., Instantaneous Bethe-Salpeter equation: Improved analytical solution

Lucha, W., see Hall

Ludwig, G., see Edgar

Lunardi, J.T., Pimentel, B.M.,
A17 (2002) 4871

A17 (2002) 4647

A17 (2002) 2776

A17 (2002) 4317

A17 (2002) 1817

A17 (2002) 790

A17 (2002) 1003

A17 (2002) 761

A17 (2002) 1003

A17 (2002) 966

A17 (2002) 2701

A17 (2002) 435

A17 (2002) 993

A17 (2002) 1183

A17 (2002) 555

A17 (2002) 555

A17 (2002) 2501

A17 (2002) 2767

A17 (2002) 2752

A17 (2002) 3607

A17 (2002) 1435

A17 (2002) 4669

A17 (2002) 4543

A17 (2002) 2555

A17 (2002) 625

A17 (2002) 4801

A17 (2002) 2333

A17 (2002) 1931

A17 (2002) 2757
Valverde, J.S. \& Manzoni, L.A., Duffin-Kemmer-Petiau theory in the causal approach Lunardi, J.T., see Casana

Luo, W., see Milton

Lydia, I.-F., Results on CP violation from the NA48 experiment at CERN

MacAlevey, P., see Robinson

MacCallum, M.A.H., Computer algebra in general relativity

Maceda, M., see Grosse

Macfarlane, A.J., The sphere $S^{6}$ viewed as a $G_{2} / \mathrm{SU}(3)$ coset space

A17 (2002) 205

A17 (2002) 4197

A17 (2002) 732

A17 (2002) 3012

A17 (2002) 2733

A17 (2002) 2707

A17 (2002) 2095

A17 (2002) 2595

Machet, B., Mass matrices and eigenstates for scalars/ pseudoscalars; indirect $\mathrm{CP}$ violation, mass hierarchies and symmetry breaking

Madore, J., see Grosse

A17 (2002) 3839

A17 (2002) 2095

Magierski, P., Bulgac, A. \& Heenen, P.-H., Neutron stars and the fermionic Casimir effect

A17 (2002) 1059

Mahapatra, D.P., Mohanty, B. \& Phatak, S.C., Acceptance dependence of fluctuation in particle multiplicity

Maia Neto, P., see Reynaud

Maia, A., see Bandeira

Maia, J.M.F., see Maia

Maia, M.D., Monte, E.M. \& Maia, J.M.F., On Friedmann's equation in brane-worlds

Maia, M.D., see Monte

Maiani, L., LHC machine program

Majumdar, P. \& Sharatchandra, H.S., Duality transformation for $(3+1)$-dimensional Yang -Mills theory

Malinin, A., see Choutko

Mallik, S., see Bagchi

Mandal, B.P., see Joglekar

Manssur, L.R.U., Nogueira, A.L.M.A. \& Santos, M.A., An extended Abelian ChernSimons model and the symplectic projector method

Manzoni, L.A., see Lunardi

Marachevsky, V.N., Casimir energy of a dilute dispersive dielectric ball: Realistic microscopic model
A17 (2002) 175

A17 (2002) 675

A17 (2002) 1003

A17 (2002) 4413

A17 (2002) 4341

A17 (2002) 4341

A17 (2002) 4355

A17 (2002) 3459

A17 (2002) 1817

A17 (2002) 51

A17 (2002) 1279

A17 (2002) 1919

A17 (2002) 205

A17 (2002) 786 
Marmo, G., see Ercolessi

Martin, J., see Brandenberger

Martín Cruz, A., see Rodríguez Querts

Masiero, A. \& Pascoli, S., Neutrinos as dark matter candidates

Matias, J., see Burgess

Mauro, D., On Koopman-von Neumann waves

Mavromatos, N.E., see Diamandis

Mavromatos, N.E., see Diamandis

Mbelek, J.P. \& Lachièze-Rey, M., A five dimensional model of effective gravitational and fine-structure constants

McKay, D.W., see Jain

McKeon, D.G.C., Observations on a $\mathrm{U}(1) \times \mathrm{U}(1)$ vector theory

Melanson, J., see Gauthier

Melnikov, V. N., Time variations of $G$ in different models

Mena, F.C., Tavakol, R. \& Bruni, M., Second order perturbations of flat dust FLRW universes with a cosmological constant

Méndez, F., see Gamboa

Mesref, L., The Jordanian $U_{h}(2)$ Yang-Mills theory

Mignemi, S., Primary scalar hair of black holes in effective string theory

Mikhailov, V.V., Low energy electron and positron spectra in the earth orbit measured by MARIA-2 instrument

Milano, L., see Calloni

Miller, J.P., The muon anomaly: Experiment and theory

Milton, K.A. \& Solovtsova, O.P., Perturbative expansions in the inclusive decay of the tau-lepton

Milton, K.A., Kalbfleisch, G.R., Luo, W. \& Gamberg, L., Theoretical and experimental status of magnetic monopoles

Minkevich, A.V., Regular inflationary cosmological models in general relativity theory
A17 (2002) 3779

A17 (2002) 3663

A17 (2002) 561

A17 (2002) 1723

A17 (2002) 1841

A17 (2002) 1301

A17 (2002) 2241

A17 (2002) 4567

A17 (2002) 4317

A17 (2002) 533

A17 (2002) 2211

A17 (2002) 2761

A17 (2002) 4325

A17 (2002) 4239

A17 (2002) 2555

A17 (2002) 4777

A17 (2002) 2768

A17 (2002) 1695

A17 (2002) 804

A17 (2002) 3318

A17 (2002) 3789

A17 (2002) 732 and gauge theories of gravitation

A17 (2002) 4441

Misra, A., Noncommutative $N=2 p-p^{\prime}$ system

A17 (2002) 1117

A17 (2002) 675

Mohanty, B., see Mahapatra

Mohideen, U., see

Klimchitskaya

Mohideen, U., see Chen

Moniz, P.V., Spherically symmetric gravitational fields: Black holes and midisuperspace quantization near the apparent horizon

Monte, E.M. \& Maia, M.D., On Schwarzschild's topology in brane-worlds

Monte, E.M., see Maia

Montvay, I., Supersymmetric Yang-Mills theory on the lattice

Morandi, G., see Ercolessi

Morgan, M.J., see Thatcher

Morris, T.R., see Arnone

Morselli, A., Dark matter search with gamma rays: the experiments EGRET and GLAST

Mostepanenko, V.M., Experimental status of corrections to Newtonian gravitation inspired by extra dimensions

Mostepanenko, V.M., Constraints on forces inspired by extra dimensional physics following from the Casimir effect

Motavali, H. \& Golshani, M., Exact solutions for cosmological models with a scalar field

Motavali, H., see Fakhri

Mukhopadhyay, S., see Bhattacharyya

A17 (2002) 4143

A17 (2002) 711

A17 (2002) 2459

A17 (2002) 4355

A17 (2002) 4341

A17 (2002) 2377

A17 (2002) 3779

A17 (2002) 1953

A17 (2002) 2283

A17 (2002) 1829

A17 (2002) 4307

A17 (2002) 722

Müller, D. \& Fagundes, H.V., Casimir energy density in closed hyperbolic universes

Mülsch, D., see Geyer

Murayama, H., Theory of neutrino masses and mixings

Murchadha, N.Ó., Constrained Hamiltonians and localsquare-root actions

Naculich, S.G., Schnitzer, H.J. \& Wyllard, N., 1/N corrections to anomalies and the AdS/CFT correspondence for
A17 (2002) 375

A17 (2002) 4081

A17 (2002) 4647

A17 (2002) 4385

A17 (2002) 4425

A17 (2002) 3403

A17 (2002) 2717 
orientifolded $\mathcal{N}=2$ orbifold and $\mathcal{N}=1$ conifold models

Nakanishi, N., see Abe

Nash, J., Babar B decay results

Nason, P., QCD at high energy

Navarro, G.A., see Daleo

Nepomechie, R.I., Supersymmetry in the boundary tricritical Ising field theory

Nersessian, A., see Geyer

Nesterenko, V.V., Lambiase, G. \& Scarpetta, G., Casimir energy of a dilute dielectric ball at zero and finite temperature

Neubert, M., Theory of CP violation in the B-meson system

Neves, C. \& Wotzasek, C., Hidden symmetries of constrained systems

Neves, R., see Kubyshin

Nešić, L.D., see Djordjević

Nichols, A., see Kogan

Nielsen, H.B., Pallua, S. \& Prester, P., Supersymmetry: A consequence of smoothness?

Nieto, M.M. \& Truax, D.R., Schrödinger equations with time-dependent $P^{2}$ and $X^{2}$ terms

Niță, B., see Robinson

Noda, H., see Shao

Nogueira, A.L.M.A., see Manssur

Nojiri, S., Odintsov, S.D. \& Ogushi, S., FriedmannRobertson-Walker brane cosmological equations from the five-dimensional bulk (A)dS black hole

Nolan, B.C., Weak solutions for weak singularities

Nordtvedt, K., Space-time variation of physical constants and the equivalence principle

Novak, J., see Gourgoulhon

Novello, M., Effective geometry in nonlinear electrodynamics

Nurowski, P., Conformal connection and equivalence problem for third order ODEs

Obers, N.A., see Halpern

Obukhov, Y.N., see Hehl

Oda, I., Higgs mechanism in the
A17 (2002) 2567

A17 (2002) 1491

A17 (2002) 2982

A17 (2002) 3123

A17 (2002) 269

A17 (2002) 3809

A17 (2002) 1183

A17 (2002) 790

A17 (2002) 2936

A17 (2002) 4025

A17 (2002) 4871

A17 (2002) 1413

A17 (2002) 2615

A17 (2002) 2073

A17 (2002) 1559

A17 (2002) 2733

A17 (2002) 615

A17 (2002) 1919

A17 (2002) 4809

A17 (2002) 2769

A17 (2002) 2711

A17 (2002) 2762

A17 (2002) 4187

A17 (2002) 2770

A17 (2002) 3897

A17 (2002) 2695 presence of a topological term

A17 (2002) 89

Odintsov, S.D., see Nojiri

A17 (2002) 4809

Odyniec, G., Quark gluon plasma - recent advances

Ogushi, S., see Nojiri

A17 (2002) 3107

A17 (2002) 4809

Olsen, S.L., Observation of large $C P$ violation in the $B$ meson system

A17 (2002) 2926

Özer, H.T., On the super field realization of super-Casimir $\mathcal{W} \mathcal{A}_{n}$ algebras

A17 (2002) 317

Pal, S.S., Derivative corrections to Dirac-Born-Infeld and Chern-Simon actions from noncommutativity

Pallua, S., see Nielsen

Panasyuk, M.I., The trapped anomalous component of the cosmic rays: The short overview of experiments

Papantonopoulos, E., see Diamandis

Papantonopoulos, E., see Diamandis

Pappa, I., see Diamandis

Paredes, A., see Barbón

Parentani, R., What did we learn from studying acoustic black holes?

Park, J.H., see Song

Pascoli, S., see Masiero

Pasupathy, J., see Ananthanarayan

Patgiri, M., see Singh

Pavlov, Yu.V., Creation of the nonconformal scalar particles in nonstationary metric

Pavlov, Yu.V., see Grib

Pérez Rojas, H., see Rodríguez Querts

Pestieau, J. \& Smith, C., A new basis for QED bound state computations

Pestieau, J., Smith, C. \& Trine, S., Positronium decay: Gauge invariance and analyticity

Puetzfeld, D., A non-standard cosmological model

Phatak, S.C., see Mahapatra

Picca, D., see Brautti

Picca, D., see Brautti

Pimentel, B.M., see Lunardi

Pimentel, B.M., see Casana

Piran, T., Gamma-ray bursts
A17 (2002) 1253

A17 (2002) 2073

A17 (2002) 1705

A17 (2002) 2241

A17 (2002) 4567

A17 (2002) 2241

A17 (2002) 3589

A17 (2002) 2721

A17 (2002) 259

A17 (2002) 1723

A17 (2002) 335

A17 (2002) 3629

A17 (2002) 1041

A17 (2002) 4435

A17 (2002) 561

A17 (2002) 4113

A17 (2002) 1355

A17 (2002) 2772

A17 (2002) 675

A17 (2002) 1111

A17 (2002) 327

A17 (2002) 205

A17 (2002) 4197

A17 (2002) 2727 
Piwnicki, P., Geometrical approach to light in inhomogeneous media

Pletnev, N.G., see Banin

Plyaskin, V., Simulation of particle fluxes in the Earth's vicinity

Pohl, M., Pulsars, blazars and dark matter with AMS

Pokorski, S., see Chankowski

Ponce, W.A., Flórez, J.B. \& Sánchez, L.A., Analysis of $\mathrm{SU}(3)_{c} \otimes \mathrm{SU}(3)_{L} \otimes \mathrm{U}(1)_{X}$ local gauge theory

Popp, L., see Bichl

Pospelov, M., see Burgess

Potting, R., see Kubyshin

Pravda, V. \& Pravdová, A., On boost-rotation symmetric space-times with (non)spinning sources

Pravdová, A., see Pravda

Prester, P., see Nielsen

Produit, N., Integral: A gammaray observatory

Quesne, C., see Bagchi

Radu, E., see van der Bij

Ragoucy, E., Quantum group symmetry of integrable systems with or without boundary

Rajantie, A., Formation of topological defects in gauge field theories

Rajeev, S.G., see Akant

Ralston, J.P., see Jain

Randall, L., Superstrings, duality, large extra dimensions

Randhawa, M. \& Gupta, M., Probing CKM parameters through unitarity, $\varepsilon_{K}$ and $\Delta m_{B_{d, s}}$

Rao, N.A., Kagali, B.A. \& Sivramkrishna, V., Bound states of Klein-Gordon particles in scalar screened Coulomb potential

Ravndal, F. \& Sundberg, M., Graviton-photon conversion on spin 0 and $1 / 2$ particles

Rebouças, M.J., see Gomero

Rego-Monteiro, M.A. \& Curado, E.M.F., Construction of a non-standard quantum field
A17 (2002) 1543

A17 (2002) 825

A17 (2002) 1733

A17 (2002) 1809

A17 (2002) 575

A17 (2002) 643

A17 (2002) 2219

A17 (2002) 1841

A17 (2002) 4871

A17 (2002) 2771

A17 (2002) 2771

A17 (2002) 2073

A17 (2002) 1787

A17 (2002) 51

A17 (2002) 1477

A17 (2002) 3649

A17 (2002) 1

A17 (2002) 2413

A17 (2002) 533

A17 (2002) 3499

A17 (2002) 1399

A17 (2002) 4793

A17 (2002) 3963

A17 (2002) 4261 theory using generalized Heisenberg algebra

Reuter, M., see Lauscher

Reynaud, S., Lamine, B., Lambrecht, A., Maia Neto, P. \& Jaekel, M.-T., Decoherence and gravitational backgrounds

Reynaud, S., see Genet

Riazuddin, Al-Aithan, T.A. \& Gilani, A.H.S., Form factors for $B \rightarrow \pi l v$ decay in a model constrained by chiral symmetry and quark model

Ringeval, C., Fermionic currents along cosmic strings

Ritus, V.I., Vacuum-vacuum amplitudes in the theory of quantum radiation by mirrors in 1+1-space and charges in $3+1$-space

Rivelles, V.O., see Girotti

Robinson, I., Downes, P., MacAlevey, P. \& Niţă, B., Approximate solutions of types $(3,1)$ and (4)

Rodríguez-Martínez, M., Brane cosmology with a bulk scalar field

Rodríguez Querts, E., Martín Cruz, A. \& Pérez Rojas, H., Effect of a magnetic field on the broken electroweak symmetry

Rojas, E., see Cordero

Rojas, J.C., see Gamboa

Romero, C., The embedding of the spactime in five dimensions

Rosa, L., see Calloni

Roudeau, P., Tau and charm physics highlights

Roy, A., see Chen

Roy, S.M., see Balachandran

Rubilar, G.F., see Hehl

Ruivo, M.C., see Jaminon

Saaidi, Kh. \& Setare, M.R., More on phase structure of nonlocal 2D generalized Yang -Mills theories (nlgYM 2 's)

Sachrajda, C.T., Phenomenology from lattice QCD

Sadooghi, N., see Ardalan

Sakai, N. \& Sugisaka, R., Low energy theorem for
A17 (2002) 1003

A17 (2002) 761

A17 (2002) 661

A17 (2002) 993

A17 (2002) 4927

A17 (2002) 2773

A17 (2002) 1033

A17 (2002) 1503

A17 (2002) 2733

A17 (2002) 2774

A17 (2002) 561

A17 (2002) 73

A17 (2002) 2555

A17 (2002) 4287

A17 (2002) 804

A17 (2002) 3037

A17 (2002) 711

A17 (2002) 4007

A17 (2002) 2695

A17 (2002) 4903

A17 (2002) 3641

A17 (2002) 3140

A17 (2002) 123 
SUSY breaking with gauge supermultiplets

A17 (2002) 4697

Sala, P.R., see Battistoni

Salesi, G., Non-Newtonian mechanics

Saliu, S.O., see Bizdadea

Samsonov, B.F., see Debergh

Sánchez, L.A., see Ponce

Santos, F.C., see Farina

Santos, M.A., see Manssur

Sanuki, T., Review of balloons muon measurement in the atmosphere

Sassot, R., see Daleo

Scarpetta, G., see Nesterenko

Schnitzer, H.J., see Naculich

Schöberl, F.F., see Hall

Schöberl, F.F., see Lucha

Schubert, Ch., see Dunne

Schützhold, R., On the cosmological constant and the cosmic coincidence problem

Schweda, M., see Bichl

Segal, A.Yu. \& Sibiryakov, A.G., Explicit $N=2$ supersymmetry for higher-spin fields in $D=4$ AdS superspace

Senovilla, J.M.M., Causal tensors and Rainich's conditions

Setare, M.R., see Saaidi

Shaisultanov, R., Vacuum polarization in QED with world-line methods

Shao, C.G., see Shao

Shao, D., Noda, H., Shao, L. \& Shao, C.G., Diffeomorphism, Hamiltonian constraints and Mandelstam identities over extended knot families $\left\{\psi_{i}\right\}_{2}^{2}$ and $\left\{\psi_{i}\right\}_{3}^{4}$

Shao, L., see Shao

Sharatchandra, H.S., see Majumdar

Sharif, M., Energy and momentum of a class of rotating gravitational waves

Shifman, M., see Gabadadze

Shirzad, A. \& Loran, F., Gauge fixing in the chain-by-chain method

Shirzad, A., see Loran

Shovkovy, I.A., Collective modes in color superconducting matter
A17 (2002) 1743

A17 (2002) 347

A17 (2002) 2191

A17 (2002) 1577

A17 (2002) 643

A17 (2002) 794

A17 (2002) 1919

A17 (2002) 1635

A17 (2002) 269

A17 (2002) 790

A17 (2002) 2567

A17 (2002) 1931

A17 (2002) 2333

A17 (2002) 956

A17 (2002) 4359

A17 (2002) 2219

A17 (2002) 1207

A17 (2002) 3641

A17 (2002) 960

A17 (2002) 615

A17 (2002) 615

A17 (2002) 175

A17 (2002) 1175

A17 (2002) 3689

A17 (2002) 4801

A17 (2002) 625

A17 (2002) 904
A17 (2002) 2775

A17 (2002) 615
Sibiryakov, A.G., see Segal

Silva, E.A., see Abreu

Simeone, C., see Giribet

Singh, N.N. \& Patgiri, M., Bimaximal mixings fron the texture of the right-handed Majorana neutrino mass matrix

Sirendaoreji \& Jiong, S., Hamiltonian structures for the nonregular constrained flows of the AKNS hierarchy

Sivakumar, M., see Harikumar Sivramkrishna, V., see Rao

Škovrlj, Lj. \& Ivezić, T., About the simultaneous co-existence of instantaneous and retarded interactions in classical electrodynamics

Smith, C., see Pestieau

Smith, C., see Pestieau

Šnobl, L. \& Hlavatý, L., Classification of six-dimensional real Drinfeld doubles

Solovtsova, O.P., see Milton

Song, D.-Y. \& Park, J.H., Coherent states and geometric phases in the CalogeroSutherland model

Spindel, Ph., see Fabris

Spinelly, J. \& Bezerra de Mello, E.R., Vacuum polarization by a magnetic field in the cosmic string spacetime

Steinacker, H., see Grosse

Steinbauer, R. \& Kunzinger, M., Generalised pseudo-Riemannian geometry for general relativity

Stösslein, U., Polarized structure functions and spin physics

Strumia, A. \& Vissani, F., Massive neutrinos and theoretical developments

Sucu, Y. \& Unal, N., Solution of massless spin one wave equation in Robertson-Walker space-time

Sugisaka, R., see Sakai

Sukhanov, S.A., see Bytsenko

Sun, X.-P. \& Weber, H.J., Melosh construction of relativistic three-quark baryon wave functions

Sundberg, M., see Ravndal
A17 (2002) 1207

A17 (2002) 395

A17 (2002) 2885

A17 (2002) 3629

A17 (2002) 2897

A17 (2002) 405

A17 (2002) 4793

A17 (2002) 2513

A17 (2002) 1355

A17 (2002) 4113

A17 (2002) 4043

A17 (2002) 3789

A17 (2002) 259

A17 (2002) 2758

A17 (2002) 4375

A17 (2002) 2095

A17 (2002) 2776

A17 (2002) 3220

A17 (2002) 1755

A17 (2002) 1137

A17 (2002) 4697

A17 (2002) 4167

A17 (2002) 2535

A17 (2002) 3963 
Sushkov, S.V., New form of the renormalization counterterms for a scalar field

Suzuki, N., Deviation from the thermal equilibrium of the accelerated detektor due to the field theoretical divergence

Svaiter, N.F., see Ford

Sýkora, T., see Iorio

Taha, M.O., see Al-Naghmoush

Tajima, H., Belle $B$ physics results

Takata, H., see Bal

Tavakol, R., see Mena

Tavakol, R., see Gomero

Tavakol, R., see Huey

Tavani, M., The AGILE Mission and gamma-ray astrophysics

Teixeira, R.G., see Casana

Teyssandier, P., Non-minimal coupling, variable speed of light and cosmology

Thatcher, M.J. \& Morgan, M.J., Birefringent electroweak defects

Thorn, C.B., see Halpern

Thorwart, J. \& Halliwell, J.J., Timeless properties for quantum cosmological models

Tighe, J.F., see Arnone

Tkachov, F.V., A theory of jet definition

Tlyachev, V.B., see Bagrov

Tort, A.C., see Farina

Tossa, J., see Batista

Trautman, A., Robinson manifolds and the shear-free condition

Triay, R., The cosmological doubt

Trine, S., see Pestieau

Truax, D.R., see Nieto

Tsuboi, Z., A note on the $\operatorname{osp}(1 \mid 2 s)$ thermodynamic Bethe ansatz equation

Turner, M.S., The new cosmology

Ullio, P., Searches for dark matter particles through cosmic ray measurements

Unal, N., see Sucu

Uraltsev, N., see Bigi

Usha Devi, A.R., Spin distributions for bipartite quantum systems
A17 (2002) 820

A17 (2002) 1026

A17 (2002) 4393

A17 (2002) 2369

A17 (2002) 685

A17 (2002) 2967

A17 (2002) 2445

A17 (2002) 4239

A17 (2002) 4261

A17 (2002) 4403

A17 (2002) 1799

A17 (2002) 4197

A17 (2002) 2777

A17 (2002) 1953

A17 (2002) 1517

A17 (2002) 2778

A17 (2002) 2283

A17 (2002) 2783

A17 (2002) 1045

A17 (2002) 794

A17 (2002) 2749

A17 (2002) 2735

A17 (2002) 4209

A17 (2002) 1355

A17 (2002) 1559

A17 (2002) 2351

A17 (2002) 3446

A17 (2002) 1777

A17 (2002) 1137

A17 (2002) 4709

A17 (2002) 2267
Uzan, J.-P., Simulated gravity in asymmetric braneworld scenarios

Vacchini, B., see Lanz

Valent, G., see Casteill

Valverde, J.S., see Lunardi

Van den Bossche, B., see Debergh

A17 (2002) 2739

A17 (2002) 435

A17 (2002) 2754

A17 (2002) 205

A17 (2002) 1577

A17 (2002) 2755

van den Hoogen, R.J., see Coley van der Bij, J.J. \& Radu, E., On rotating regular non-Abelian solutions

A17 (2002) 1477

van Holten, J.W., World-line deviations and epicycles

Vickers, J.A., Weak singularities in general relativity

Villain, L. \& Bonazzola, S., Numerical time evolution of inertial modes in slowly rotating neutron stars

Villalba, V.M., Creation of scalar and Dirac particles in the presence of a time varying electric field in an anisotropic universe

Villarreal, C., Esquivel-Sirvent, R. \& Cocoletzi, G.H., Modification of Casimir forces due to band gaps in periodic structures

Visinescu, M., Fermions in Taub-NUT background

Vissani, F., see Strumia

Volovich, I.V., see Djordjević

von Borzeszkowski, H.-H., Teleparallelized and affine theories of gravity: New perspectives for Machian and quantum gravity

Vytheeswaran, A.S., Hidden symmetries in second-class constrained systems: Are new fields necessary?

A17 (2002) 2764

A17 (2002) 2779

A17 (2002) 2780

A17 (2002) 2781

A17 (2002) 798

A17 (2002) 1049

A17 (2002) 1755

A17 (2002) 1413

A17 (2002) 4153

A17 (2002) 4095

Wahsner, R., Mach's philosophy, his criticism of the Newtonian space-time conception and the possibility of a deductive cosmology

Weber, H.J., see Sun

Weigel, H., see Graham

Wickramasekara, S., see Kaldass

Winstanley, E., Is there classical super-radiance on KerrNewman-anti-de Sitter black holes?
A17 (2002) 2782

A17 (2002) 4245

A17 (2002) 2535

A17 (2002) 846
A17 (2002) 3749 
Wise, M.B., Symmetries for strongly interacting heavy particles

A17 (2002) 1199

Wise, M.B., Recent progress in heavy quark physics

Wotzasek, C., see Neves

Wu, Y.-F., see Liao

Wulkenhaar, R., see Bichl

Wyllard, N., see Naculich

Yanovski, A.B., Bundles of Lie algebras and compatible Poisson brackets

A17 (2002) 946

Yasuè, M., see Kitabayashi

Zachos, C., Deformation quantization: Quantum mechanics lives and works in phasespace

A17 (2002) 3068

A17 (2002) 4025

A17 (2002) 4669

A17 (2002) 2219

A17 (2002) 2567

A17 (2002) 2519

A17 (2002) 297

Zait, R.A., Soliton solutions in a modified $\phi^{4}$ model

Zakharov, V.I., see Gubarev

Zaslavskii, O.B., 2D dilaton gravity and regular horizons away from the Hawking temperature

A17 (2002) 1013
Zhang, K.-S., Chen, G. \& Liu, L.-S., A Monte Carlo study on the dynamical fluctuations inside quark and antiquark jets

A17 (2002) 4543

Zhang, Q.H., Ф-Measure of pions in high energy heavyion collisions

A17 (2002) 109

Zhao, W.-Y. \& Khanna, F.C., Screening length, dispersion relations and quark potential in thermo field dynamics

A17 (2002) 2911

Zhuang, P.-F., see Chen

A17 (2002) 1435

Zhukovsky, V.C., Regular and stochastic configurations of gauge fields with nontrivial topology

A17 (2002) 914

Zuccon, P., A Monte Carlo simulation of the cosmic rays interactions with the Earth's atmosphere

A17 (2002) 1625

Zwirner, F., The Higgs puzzle:

Experiment and theory

A17 (2002) 3300

Życzkowskí, K., see Bengtsson 